Research Paper

\title{
Long non-coding RNA CDKN2B antisense RNA 1 gene inhibits Gemcitabine sensitivity in bladder urothelial
} carcinoma

\author{
Dalong Xie ${ }^{1}$, Hui Zhang2, Chao Shang ${ }^{3 凶}$ \\ 1. Department of Anatomy, College of Basic Medicine, China Medical University, Shenyang, 110001, China \\ 2. Department of Urinary surgery, Shengjing Hospital, China Medical University, Shenyang, 110004, China \\ 3. Department of Neurobiology, College of Basic Medicine, China Medical University, Shenyang, 110001, China \\ $\square$ Corresponding author: Chao Shang, Department of Neurobiology, China Medical University, No.77 Puhe Road, Shenyang North New Area, Shenyang, \\ 110122, China. Phone/Fax: +86-24-23256666; E-mail address: chaos@cmu.edu.cn \\ (C) Ivyspring International Publisher. This is an open access article distributed under the terms of the Creative Commons Attribution (CC BY-NC) license \\ (https://creativecommons.org/licenses/by-nc/4.0/). See http://ivyspring.com/terms for full terms and conditions.
}

Received: 2018.01.30; Accepted: 2018.04.15; Published: 2018.05.25

\begin{abstract}
Objective: To investigate the clinical significance of long noncoding RNA (IncRNA) CDKN2B antisense RNA 1 (CDKN2B-AS) gene and its effects on Gemcitabine sensitivity in BUC.

Materials and Methods: The expression of CDKN2B-AS gene was examined with real-time quantitative PCR. The cell proliferation and the half maximal inhibitory concentration (IC50) of Gemcitabine were detected with enhanced CCK-8 assay. The apoptosis rate was examined using Annexin V-FITC/PI double-staining apoptosis kit. The protein expression was examined with western blotting. The activity of Wnt signaling pathway was examined with TOP/FOP luciferase assay.

Results: CDKN2B-AS gene was high-expressed in BUC tissues and J82, T24 cells compared with paracancerous normal urothelial tissues and SV-HUC-1 cells. Furthermore, the high-expression of CDKN2B-AS gene was related with high pathological grade and low Gemcitabine sensitivity of BUC tissues. The expression of CDKN2B-AS gene in Gemcitabine-resistant T24/Gem cells was much higher than that in T24 cells. Knockdown of CDKN2B-AS gene sensitized T24/Gem cells to Gemcitabine, promoted Gemcitabine-induced cytotoxicity. Knockdown of CDKN2B-AS gene inactivated Wnt signaling pathway, and Wnt signaling pathway mediated the effects on Gemcitabine sensitivity induced by CDKN2B-AS knockdown in T24/Gem cells.

Conclusion: LncRNA CDKN2B-AS is high-expressed in BUC and related to low Gemcitabine sensitivity of BUC. CDKN2B-AS inhibited Gemcitabine sensitivity through Wnt signaling pathway in BUC.
\end{abstract}

Key words: long noncoding RNA; bladder urothelial carcinoma; CDKN2B antisense RNA 1; Wnt signaling pathway; chemotherapy

\section{Introduction}

Bladder cancer is a malignant neoplasm originating from bladder mucosa and is one of the most common malignant neoplasms in genitourinary system worldwide [1]. The pathological types of bladder cancer mainly include bladder urothelial carcinoma (BUC), bladder adenocarcinoma and bladder squamous cell carcinoma. BUC is the most common pathological type of bladder cancer, named bladder transitional cell carcinoma before, accounting for more than $90 \%$ of the total number of patients with bladder cancer [2].

Chemotherapy is one of the major therapeutic methods for BUC, which can significantly reduce the metastasis and recurrence of BUC, and improve the prognosis of BUC. However, chemotherapeutic resistance reduces the sensitivity of BUC to 
chemotherapeutic drugs and contributes a barrier leading to treatment failure $[3,4]$. Therefore, it is important and necessary to elucidate the underlying mechanism of BUC chemotherapeutic drug resistance and find new therapeutic targets.

In recent years, long noncoding RNA (lncRNA) has become a hotspot in the research of life science, especially in oncology. As the progresses of functional studies, lncRNAs have been found to be involved in a variety of human complex diseases, including tumors [5-7]. Accumulating evidences prove lncRNAs play important roles in carcinogenesis by participating in various important cytobiological behaviors, including cell cycle, apoptosis, invasion, autophagy and so on [8-10]. Recent studies reported some lncRNAs could be biomarkers for diagnosis and prognosis as well as therapeutic targets of tumors [11-13].

CDKN2B antisense RNA 1 (CDKN2B-AS) gene is a lncRNA gene identified by Pasmant $\mathrm{E}$ in the genetic study of a melanoma-neural system tumor family in 2007, which also named Antisense Noncoding RNA In The INK4 Locus (ANRIL) [14]. Recent studies had found that the CDKN2B-AS gene up-regulated in several malignant tumors, such as hepatocellular carcinoma, nasopharyngeal carcinoma and gallbladder cancer, and acted as an oncogene [15-17]. Zhu H reported that CDKN2B-AS was overexpressed in bladder cancer, and regulated bladder cancer cell proliferation and apoptosis [18], which suggested that CDKN2B-AS might facilitate the tumorigenesis of BUC. However, the function and underlying mechanisms of CDKN2B-AS on chemotherapeutic resistance are still unclear.

\section{Materials and methods}

\section{Clinical specimens}

In this study, 81 BUC patients were hospitalized and treated in the Department of Urology of Shengjing Hospital from September 2013 to February 2015. BUC tissue specimens were obtained through cystoscopy. The normal urothelial tissue (NUT) specimens were obtained from 34 patients with the same age period undergoing lithocystotomy, cystostomy and suprapubic transvesical prostatectomy. All tissue specimens were diagnosed by two pathologists, and the clinical data were complete. All patients were not treated with radiotherapy or chemotherapy before operation.

After cystoscopy, all BUC patients were treated with Gemcitabine. After two or three cycles of chemotherapy, all BUC patients were examined by cystoscopy and imaging tests, and the curative effects were verified according with RECIST Response Evaluation Criteria. BUC patients sensitive to
Gemcitabine were relieved completely or partially, and the conditions of patients insensitive to Gemcitabine were stable or deteriorating.

\section{Ethics}

Study data and informed consent were obtained in accordance with the Declaration of Helsinki and approved by the Ethics Committee of Shengjing Hospital. All patients were given a written explanation of the study, and they provided their written informed consent before participating.

\section{Cell lines and culture}

Human bladder epithelial immortalized cell line (SV-HUC-1) and BUC cell lines (J82, T24) were purchased from the American Type Culture Collection (Manassas, USA). Those Cells were cultured in Dulbecco's modified Eagle's medium (DMEM) containing with $10 \%$ fetal bovine serum (Thermo Fisher Scientific, USA) in a $95 \%$ air $/ 5 \% \mathrm{CO}_{2}$ incubator under $37^{\circ} \mathrm{C}$.

Gemcitabine-resistant T24/Gem cell line was set up previously through induction with low concentration of Gemcitabine in our laboratory. To maintain the resistant phenotype, T24/Gem cells was cultured with $0.5 \mu \mathrm{g} / \mathrm{mL}$ Gemcitabine (Sigma, USA), and cultured in Gemcitabine-free medium for 7 days prior to experiment.

\section{Real-time quantitative PCR}

Total RNA was isolated from tissues and cells using Trizol reagent (Thermo Fisher Scientific, USA), and reverse transcribed into cDNA using lnRcute lncRNA First-Strand cDNA Synthesis Kit (Tiangen, China). The expression of CDKN2B-AS is examined using lnRcute lncRNA qPCR Detection Kit (Tiangen, China) according to manufacturer's instructions. The primers of CDKN2B-AS were 5' - TGCTCTATCCGCC AATCAGG -3' (sense) and 5'- GGGCCTCAGTGGCA CATACC -3' (antisense) [19]. The expression of CDKN2B-AS was defined based on the threshold cycle (Ct), and relative expression levels were calculated as $2^{-\triangle \Delta C T}$ after normalization with reference to expression of $\beta$-actin. The primers of $\beta$-actin were 5'- GCACCACACCTTCTACAATGAG - $3^{\prime}$ (sense) and 5' - ACAGCCTGGATGGCTACGT -3' (antisense).

\section{Cells Transfection}

The inhibitor of CDKN2B-AS (smart silencer-CDKN2B-AS, ss-CDKN2B-AS) and its negative control (ss-NC) were designed and synthesized by the Ribobio Company (Guangzhou, China). The full sequence of CTNNB1 was cloned into pUC plasmid to construct pUC-CTNNB1 by Cyagen Biosciences Inc (Santa Clara, USA), and the pUC plasmid was used as negative control (pUC-NC). 
Transient transfection of smart silencers and plasmids were carried out using Lipofectamine ${ }^{\mathrm{TM}} 3000$ (Thermo Fisher Scientific, USA) in a 12-well culture plates following the manufacturer's instructions.

\section{Cell proliferation assay}

The cell proliferation of T24/Gem cells was examined using Enhanced Cell Counting Kit-8 (Beyotime, China). The T24/Gem cells in logarithmic growth phase were digested with trypsin, cleaned by PBS, and suspended in the culture medium. Then, 2000 cells in $100 \mu \mathrm{l}$ medium were added into one pore of 96-well plates, added $10 \mu \mathrm{l}$ enhanced CCK-8 solution to each pore, and incubated for 1 hours. The absorbance value $(450 \mathrm{~nm})$ was detected with a Microplate Reader.

\section{Apoptosis rate detection}

The apoptosis rate of $\mathrm{T} 24 / \mathrm{Gem}$ cells was detected using Annexin V-FITC/PI apoptosis detection kit (Jiancheng, China). $2 \times 10^{5}$ cells were centrifuged with $1000 \mathrm{~g}$ for 5 minutes, the supernatant was removed, and $500 \mu \mathrm{l}$ binding buffer was added to suspend cells gently. $5 \mu \mathrm{l}$ Annexin V-FITC and $5 \mu \mathrm{l}$ PI were added into cell suspension in turn and mixed gently. The cell suspension was incubated at room temperature for 10 minutes, and the apoptosis rate was detected by flow cytometry. Then, the Diva 8.0 software (BD, USA) was used to analysis the apoptosis rate by calculated the percent of cells with FITC-Annexin V positive/PI negative in the right lower quadrant.

\section{Drug sensitivity assay}

T24/Gem cells were treated with Gemcitabine $(0.1 \mu \mathrm{g} / \mathrm{mL}, 0.5 \mu \mathrm{g} / \mathrm{mL}, 1 \mu \mathrm{g} / \mathrm{mL}, 5 \mu \mathrm{g} / \mathrm{mL}, 10$ $\mu \mathrm{g} / \mathrm{mL}, 20 \mu \mathrm{g} / \mathrm{mL}$ ). The cell viability was examined 24 hours later. Then, the half maximal inhibitory concentration (IC50) of Gemcitabine was calculated according to the dose-response curve.

\section{Western Blotting}

Nuclear and Cytoplasmic Protein of T24/Gem Cells were extracted using Nuclear and Cytoplasmic Protein Extraction Kit, and quantified using Bradford Protein Assay Kit (Beyotime, China). Totally $30 \mu \mathrm{g}$ protein were processed including separated by sodium dodecyl sulfate-polyacrylamide gel electrophoresis (SDS-PAGE), transferred to a polyvinylidene fluoride (PVDF) membrane, blocked with non-fat milk, hybridized with CTNNB1 and CCND1 (Cyclin D1) antibodies (ab32572 and EPR2241, Abcam, USA) and incubated with second antibody. The PVDF membrane treated with BeyoECL Plus reagent (Beyotime, China). Then, the bands were detected and analyzed by ImageJ software (NIH, USA).

\section{Luciferase assay}

The TOP Flash and FOP FLASH luciferase reporter vectors which contain wild-type or mutant TCF binding sites were purchased from Biovector NTCC Ltd (Beijing, China). T24/Gem cells were transiently co-transfected with luciferase plasmid and ss-CDKN2B-AS vector using Lipofectamine 3000. 48 hours later, dual-lucy assay kit from Vigorous Biotech (Beijing, China) was used to quantified the activity of Wnt signaling pathway according to manufacturer's instructions [20,21]. The firefly luciferase used as a base line and renilla luciferase used as the internal control.

\section{Statistical analysis}

The SPSS 22.0 (IBM, USA) was used for statistical analysis. All data was expressed as mean \pm standard deviation and analyzed with one-way ANOVA and Student's $t$-test. If the $P$ value was less than 0.05 , the difference was statistically significant $\left({ }^{*}\right.$ and $\left.\#\right)$.

\section{Results}

\section{CDKN2B-AS gene was high-expressed in BUC}

Compared with NUT specimens, CDKN2B-AS gene was high-expressed significantly in BUC tissues specimens (Fig 1A, $P<0.05$ ). The expression of CDKN2B-AS gene in $\mathrm{J} 82$ and T24 cells was much higher than that in SV-HUC-1 cells (Fig 1B, $P<0.05$ ).
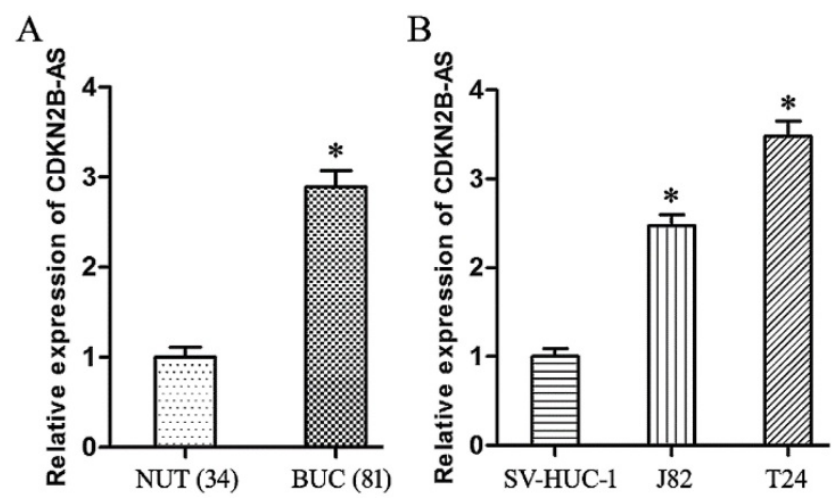

Figure 1: CDKN2B-AS gene was high-expressed in BUC. A: The expression of CDKN2B-AS gene in NUT and BUC specimens. B: The expression of CDKN2B-AS gene in SV-HUC-1, 182 and T24 cells. $* P<0.05$.

As shown in Table 1, the increased expression of CDKN2B-AS showed the positive correlation with high pathological grade of BUC $(P<0.05)$, but was not correlated with other parameters, including gender, age and smoking history of BUC patients $(P>0.05)$. These founding suggested that CDKN2B-AS gene was involved in the progress of BUC. 
Table 1. The correlation analysis between the expression of CDKN2B-AS gene and the clinicopathological factors of 81 BUC tissues

\begin{tabular}{lllll}
\hline pathological factors & & $\begin{array}{c}\text { case } \\
\text { relative expression of } \\
\text { CDKN2B-AS }\end{array}$ & $P$ \\
\hline gender & male & 52 & $2.882 \pm 0.174$ & 0.693 \\
age(years) & female & 29 & $2.911 \pm 0.213$ & \\
& $<57$ & 41 & $2.958 \pm 0.315$ & 0.072 \\
smoking history & $\geq 57$ & 40 & $2.825 \pm 0.342$ & 0.157 \\
(more than ten years) & no & 49 & $2.853 \pm 0.276$ & \multirow{2}{*}{$0.018^{*}$} \\
grade & yes & 32 & $2.951 \pm 0.337$ & \\
& low grade & 39 & $2.805 \pm 0.263$ & $<0.001^{*}$ \\
chemotherapy & high grade & 42 & $2.973 \pm 0.352$ & \\
& sensitive & 44 & $2.715 \pm 0.271$ &
\end{tabular}

Note: * indicated that the difference was statistically significant. $P<0.05$

Furthermore, the over-expression of CDKN2BAS was associated with the low sensitivity to Gemcitabine of BUC patients (Table 1, $P<0.05$ ), which preliminarily confirmed CDKN2B-AS participated in the genesis of chemotherapy resistance in BUC.

\section{High-expression of CDKN2B-AS was correlated with poor response to Gemcitabine in BUC}

As showed in Fig.2A, the IC50 of Gemcitabine in T24 and T24/Gem cells were 1.64 $\pm 0.21 \mu \mathrm{g} / \mathrm{mL}$ and
$5.92 \pm 0.36 \mu \mathrm{g} / \mathrm{mL}(P<0.05)$. T24/Gem cells showed lower sensitivity to Gemcitabine compared with T24 cells. And, the expression of CDKN2B-AS in T24/Gem cells was up-regulated compared with T24 cells (Fig.2B).

To verify the roles of CDKN2B-AS on chemotherapy resistance, T24/Gem cells were transfected with ss-CDKN2B-AS to silence the CDKN2B-AS expression (Fig.2C). And, knockdown of CDKN2B-AS decreased the IC50 of Gemcitabine from $5.85 \pm 0.33 \mu \mathrm{g} / \mathrm{mL}$ to $2.16 \pm 0.15 \mu \mathrm{g} / \mathrm{mL}$ in $\mathrm{T} 24 / \mathrm{Gem}$ cells (Fig.2D, P<0.05), which certified that CDKN2B-AS knockdown sensitized T24/Gem cells to Gemcitabine.

Moreover, under treating with Gemcitabine (0.5 $\mu \mathrm{g} / \mathrm{mL})$, knockdown of CDKN2B-AS depressed cell viability of T24/Gem cells (Fig.2E, $P<0.05$ ), and promoted apoptosis of T24/Gem cells (Fig.2F, $P<0.05)$. Knockdown of CDKN2B-AS advanced significantly Gemcitabine-induced cytotoxicity.

\section{CDKN2B-AS knockdown inactivated Wnt signaling pathway}

Luciferase assay discovered knockdown of CDKN2B-AS restrained significantly the relative
A

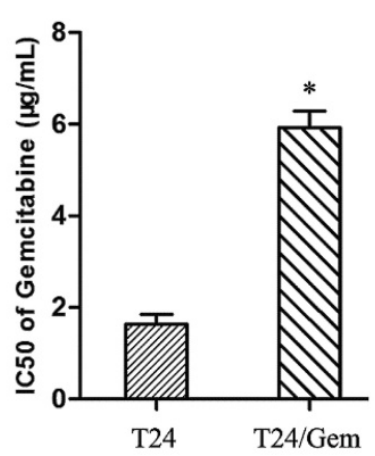

$\mathrm{D}$

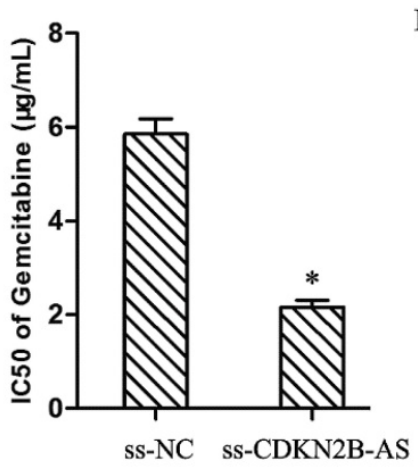

B

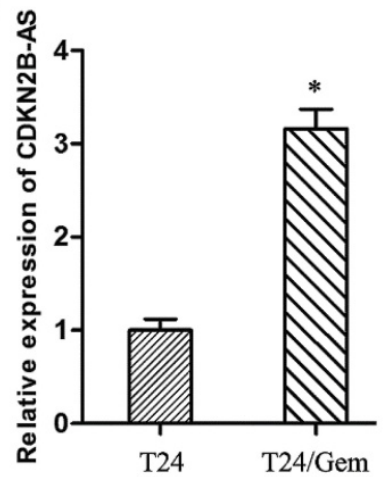

E

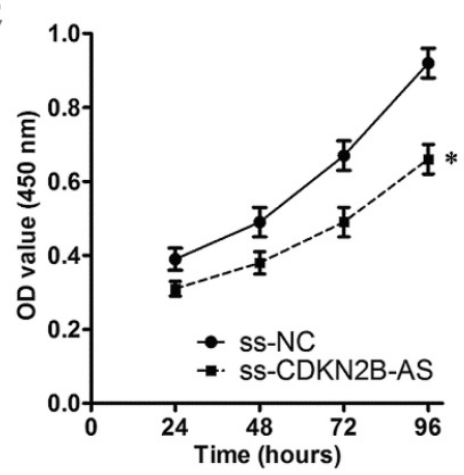

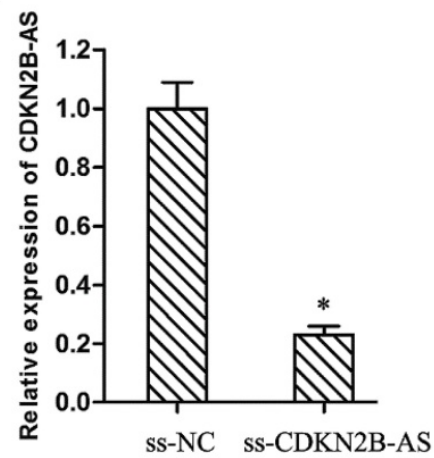

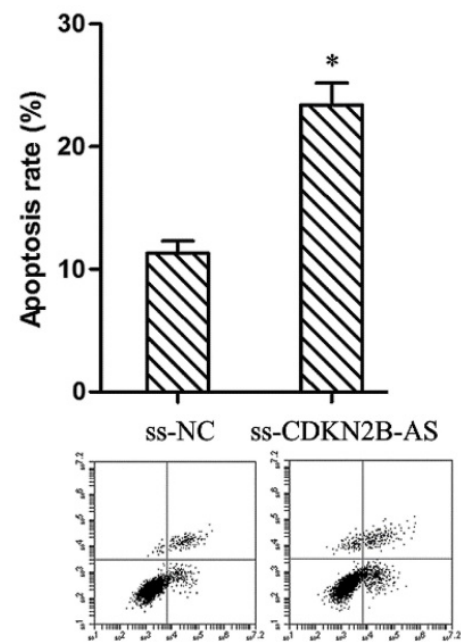

Figure 2. High-expression of CDKN2B-AS was correlated with Gemcitabine resistance of BUC. A: The IC50 of Gemcitabine in T24 and T24/Gem. B: The expression of CDKN2B-AS gene in T24 and T24/Gem cells. C: The expression of CDKN2B-AS gene in T24/Gem cells. D: The IC50 of Gemcitabine in T24/Gem cells. E: The cell viability of T24/Gem cells under treating with Gemcitabine $(0.5 \mu \mathrm{g} / \mathrm{mL})$. F: The cell apoptosis of T24/Gem cells under treating with Gemcitabine $(0.5 \mu \mathrm{g} / \mathrm{mL})$. $* P<0.05$. 


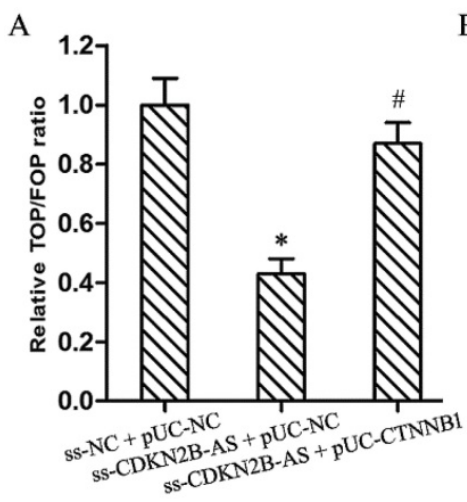

B

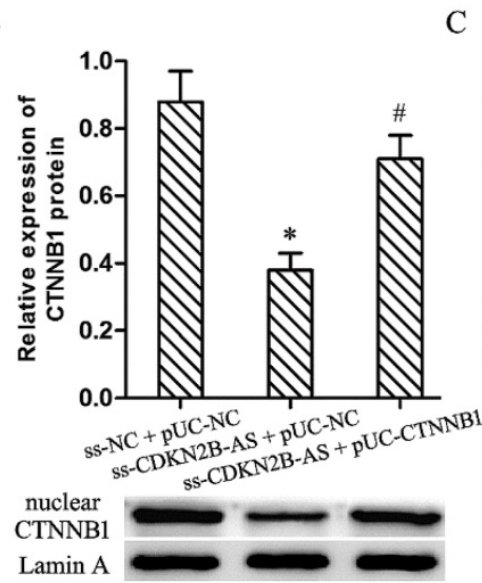

$\mathrm{C}$

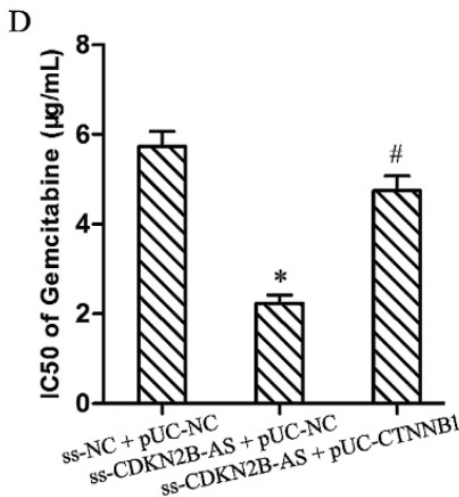

$\mathrm{E}$
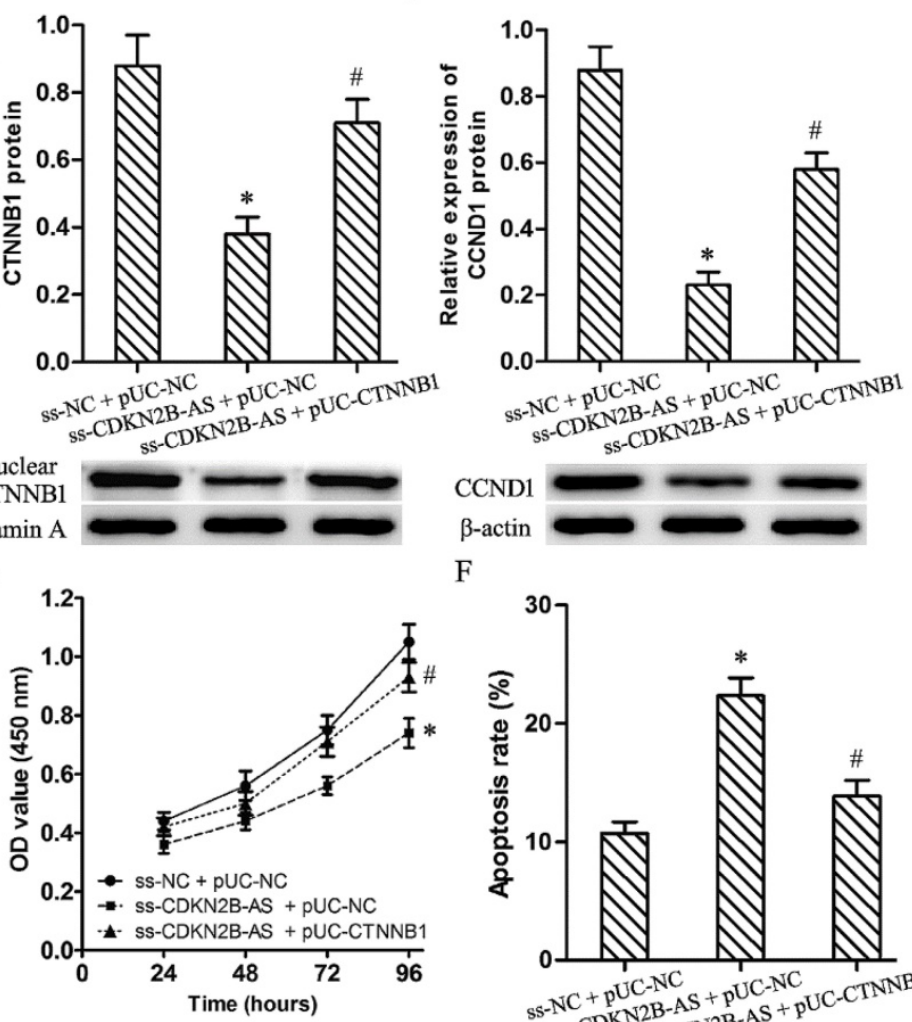

$\mathrm{F}$
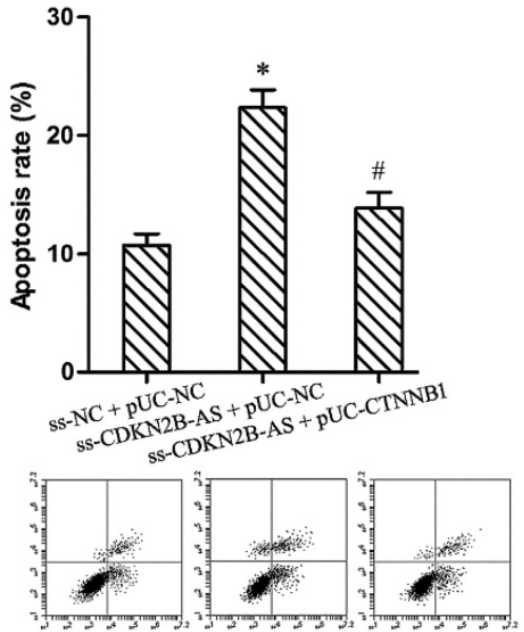

Figure 3. Wnt signaling pathway mediated the effects on Gemcitabine sensitivity induced by CDKN2B-AS knockdown in T24/Gem cells. A: The ratio of TOP/FOP luciferase values in T24/Gem cells. B: The relative expression of nuclear CTNNB1 protein in T24/Gem cells. C: The relative expression of CCND1 protein in T24/Gem cells. D: The IC50 of Gemcitabine in T24/Gem cells. E: The cell viability of T24/Gem cells under treating with Gemcitabine $(0.5 \mu \mathrm{g} / \mathrm{mL})$. F: The cell apoptosis of T24/Gem cells under treating with Gemcitabine $(0.5 \mu \mathrm{g} / \mathrm{mL}) . * P<0.05$ vs ss-NC+pUC-NC, \# $P<0.05$ vs ss-CDKN2B-AS+pUC-NC.

TOP/FOP luciferase ratio in T24/Gem cells (Fig.3A, $P<0.05)$. And, CDKN2B-AS knockdown silenced the expression of CTNNB1 (nuclear) and CCND1 protein in T24/Gem cells (Fig.3B\&3C, P<0.05). Those founding proved CDKN2B-AS knockdown inactivated Wnt signaling pathway.

\section{Wnt signaling pathway mediated the effects on Gemcitabine sensitivity induced by CDKN2B-AS knockdown in T24/Gem cells.}

Plasmid pUC-CTNNB1 was transfected into T24/Gem cells to activate Wnt signaling pathway which restrained by knockdown of CDKN2B-AS. Luciferase assay and western blotting confirmed the activating of Wnt signaling pathway (Fig.3A-3C, $P<0.05)$.

While combining use ss-CDKN2B-AS and pUC-CTNNB1, T24/Gem showed lower sensitivity to Gemcitabine when compared with ss-CDKN2B-AS + pUC-NC group. The IC50 of Gemcitabine in T24/Gem cells increased from $2.24 \pm 0.18 \mu \mathrm{g} / \mathrm{mL}$ to $4.75 \pm 0.33$ $\mu \mathrm{g} / \mathrm{mL}$ (Fig.3D, $P<0.05$ ). Moreover, under treating with Gemcitabine $(0.5 \mu \mathrm{g} / \mathrm{mL}), \quad$ CTNNB1 up-regulation advanced cell viability and depressed apoptosis rate in T24/Gem cells (Fig.3E, 3F, P<0.05).

To sum up, activating of Wnt signaling pathway restored mostly the effecting of CDKN2B-AS knockdown on Gemcitabine sensitivity in T24/Gem cells.

\section{Discussion}

Recent literatures reported lncRNAs was involved in the formation and maintenance of chemotherapeutic resistance in almost all malignant tumors. Colon Cancer Associated Transcript 1 (CCAT1) gene increased resistance to cisplatin in non-small-cell lung cancer [22]. Our previous study found Growth Arrest Specific 5 (GAS5) gene inhibited the doxorubicin resistance of bladder transitional cell carcinoma [23].

Gemcitabine is a kind of broad-spectrum antimetabolite, and a first-line chemotherapeutic drug 
for BUC. Gemcitabine is a cytosine analogue, which can replace one of the building blocks of nucleic acids, lead to "masked chain termination", inhibit further DNA synthesis, and thereby lead to cell death. However, Gemcitabine resistance is a barrier leading to treatment failure.

Firstly, CDKN2B-AS gene was high-expressed in BUC tissues and cell lines, and its over-expression was positive related with high pathological grade of BUC, which suggested that CDKN2B-AS gene was involved in the progress of BUC. Furtherly, the over-expression of CDKN2B-AS was associated with the low sensitivity to Gemcitabine of BUC patients, which preliminarily confirmed CDKN2B-AS participated in the genesis of chemotherapy resistance in BUC. $\mathrm{Xu} \mathrm{R}$ reported CDKN2B-AS contributed to paclitaxel resistance of lung adenocarcinoma A549 cells [24]. Knockdown of CDKN2B-AS inhibited the development of multidrug resistance in gastric cancer cells to paclitaxel or 5-fluorouracil [25]. Nevertheless, it is still unknown whether CDKN2B-AS take part in chemotherapeutic resistance of $\mathrm{BUC}$, especially to Gemcitabine.

To verified the roles of CDKN2B-AS on Gemcitabine resistance, the impacts of CDKN2B-AS on Gemcitabine resistance in BUC were examined by a series of loss of function assays. CDKN2B-AS knockdown decreased IC50 of Gemcitabine in T24/Gem cells and promoted the cytotoxicity induced Gemcitabine, which showed CDKN2B-AS knockdown advanced Gemcitabine sensitivity in T24/Gem cells. Nevertheless, the underlying mechanism is unknown.

It is well known that the $\mathrm{Wnt} / \beta$-catenin signaling plays significant roles in human cancers. The opening and closing of the Wnt signaling pathway directly controls many genes related to growth and metabolism. And, it crosstalks with other signaling pathways (such as TGF- $\beta$ /BMP, Hedgehog, PI3K, etc.) to influence their downstream genes. Accumulating evidence showed that abnormal expression of lncRNAs were associated with activating of Wnt signaling pathway, following modulated the chemotherapeutic resistance of cancers. For example, H19 contributed to methotrexate resistance via activating Wnt signaling pathway [25]. Our previous study revealed that knockdown of Taurine Up-Regulated 1 (TUG1) restrained the resistance of BUC to Doxorubicin through Wnt signaling pathway [20]. Moreover, Zhang H reported ANRIL suppression could inhibit Wnt signaling pathway [27].

Consequently, Wnt signaling pathway was chosen as targets to highlight the CDKN2B-AS associated Gemcitabine resistance in BUC. CTNNB1, also named $\beta$-catenin, is a crucial signaling molecule in canonical Wnt signaling pathway. CTNNB1 accumulates in the cytoplasm and then translocates to the nucleus and subsequently activates downstream target genes, such as CCND1. Then, Flash luciferase assay and western blotting confirmed CDKN2B-AS knockdown inactivated Wnt signaling pathway.

Accordingly, we speculate that knockdown of CDKN2B-AS can advance Gemcitabine sensitivity of BUC through Wnt signaling pathway. Follow up experiments verified this hypothesis, Wnt signaling pathway mainly mediated the effects on Gemcitabine sensitivity induced by CDKN2B-AS knockdown in 24/Gem cells. However, the underlying mechanism of CDKN2B-AS regulates Wnt signaling pathway remains unknown.

Recent researches revealed some "lncRNA/ microRNA" pair modulated the activating of Wnt signaling pathway to participate in the pivotal cytobiological behaviors, including chemotherapeutic resistance. Wang $\mathrm{Y}$ reported that CDKN2B-AS negatively regulated miR-let-7a to promote tumorigenicity and cisplatin resistance in nasopharyngeal carcinoma [28]. And, Let-7 could repressed self-renewal of hepatocellular cancer stem-like cells through inhibiting Wnt signaling pathway [29]. Therefore, we speculate CDKN2B-AS might sponge Let-7 to activate Wnt signaling pathway, and then participate in Gemcitabine resistance of BUC, which requires further research to confirm.

\section{Conclusions}

High-expression of lncRNA CDKN2B-AS is associated with the low sensitivity to Gemcitabine of BUC patients, CDKN2B-AS inhibits Gemcitabine sensitivity through Wnt signaling pathway in BUC. Our findings help to elucidate the underling mechanism of chemotherapeutic resistance in BUC, and future study will provide a novel therapeutic target for BUC.

\section{Acknowledgments}

This study was funded by the National Nature Science Foundation of China (No. 81172408, No. 30901480 and No.81301834).

\section{Authors' contributions}

Da-Long Xie analyzed the data and wrote the manuscript; Chao Shang designed the study and revised the manuscript; Da-Long Xie and Hui Zhang performed all the experiments and analyzed the data.

\section{Competing Interests}

The authors have declared that no competing interest exists. 


\section{References}

1. Siegel RL, Miller KD, Jemal A. Cancer statistics, 2018. CA Cancer J Clin. 2018; 68(1):7-30. doi: 10.3322/caac. 21442.

2. Martinez Rodriguez RH, Buisan Rueda O, et al. Bladder cancer: Present and future. Med Clin (Barc). 2017; 149(10):449-455. doi: 10.1016/j.medcli.2017.06.009.

3. Erlich A, Zlotta AR. Treatment of bladder cancer in the elderly. Investig Clin Urol. 2016; 57 Suppl 1: S26-35. doi: 10.4111/icu.2016.57.S1.S26.

4. Anghel RM, Gales LN, Trifanescu OG. Outcome of urinary bladder cancer after combined therapies. J Med Life. 2016; 9(1):95-100.

5. Kwok ZH, Tay Y. Long noncoding RNAs: lincs between human health and disease. Biochem Soc Trans. 2017; 45(3):805-812. doi: 10.1042/BST20160376.

6. Maoz R, Garfinkel BP, Soreq H. Alzheimer's Disease and ncRNAs. Adv Exp Med Biol. 2017; 978:337-361. doi: 10.1007/978-3-319-53889-1_18.

7. Gong W, Zheng J, Liu X, et al. Knockdown of Long Non-Coding RNA KCNQ1OT1 Restrained Glioma Cells' Malignancy by Activating miR-370/CCNE2 Axis. Front Cell Neurosci. 2017; 11:84. doi: 10.3389 /fncel.2017.00084

8. Renganathan A, Felley-Bosco E. Long Noncoding RNAs in Cancer and Therapeutic Potential. Adv Exp Med Biol. 2017; 1008:199-222. doi: 10.1007/978-981-10-5203-3_7.

9. Schmitt AM, Chang HY. Long Noncoding RNAs in Cancer Pathways. Cancer Cell. 2016; 29(4):452-463. doi: 10.1016/j.ccell.2016.03.010.

10. Ren $K, X u$ R, Huang J, et al. Knockdown of long non-coding RNA KCNQ1OT1 depressed chemoresistance to paclitaxel in lung adenocarcinoma. Cancer Chemother Pharmacol. 2017; 80(2):243-250. doi: 10.1007/s00280-017-3356-Z.

11. Zheng $\mathrm{B}$, Jeong $\mathrm{S}$, Zhu $\mathrm{Y}$, et al. miRNA and lncRNA as biomarkers in cholangiocarcinoma(CCA). Oncotarget. 2017; 8(59):100819-100830. doi: 10.18632/oncotarget.19044.

12. Chen J, Hu L, Wang J, et al. Low Expression LncRNA TUBA4B is a Poor Predictor of Prognosis and Regulates Cell Proliferation in Non-Small Cell Lung Cancer. Pathol Oncol Res. 2017; 23(2):265-270. doi: 10.1007/s12253-016-0089-y.

13. Slaby $\mathrm{O}$, Laga $\mathrm{R}$, Sedlacek $\mathrm{O}$. Therapeutic targeting of non-coding RNAs in cancer. Biochem J. 2017; 474(24):4219-4251. doi: 10.1042/BCJ20170079.

14. Pasmant E, Laurendeau I, Héron D, et al. Characterization of a germ-line deletion, including the entire INK4/ARF locus, in a melanoma-neural system tumor family: identification of ANRIL, an antisense noncoding RNA whose expression coclusters with ARF. Cancer Res. 2007; 67(8): 3963-9. doi: 10.1158/0008-5472.CAN-06-2004.

15. Huang MD, Chen WM, Qi FZ, et al. Long non-coding RNA ANRIL is upregulated in hepatocellular carcinoma and regulates cell apoptosis by epigenetic silencing of KLF2. J Hematol Oncol. 2015; 8:50. doi: 10.1186/s13045-015-0146-0

16. Zou ZW, Ma C, Medoro L, et al. LncRNA ANRIL is up-regulated in nasopharyngeal carcinoma and promotes the cancer progression via increasing proliferation, reprograming cell glucose metabolism and inducing side-population stem-like cancer cells. Oncotarget. 2016; 7(38):61741-61754. doi: 10.18632/oncotarget.11437.

17. Liu B, Shen ED, Liao MM, et al. Expression and mechanisms of long non-coding RNA genes MEG3 and ANRIL in gallbladder cancer. Tumour Biol. 2016; 37(7):9875-86. doi: 10.1007/s13277-016-4863-y.

18. Zhu H, Li X, Song $\mathrm{Y}$, et al. Long non-coding RNA ANRIL is up-regulated in bladder cancer and regulates bladder cancer cell proliferation and apoptosis through the intrinsic pathway. Biochem Biophys Res Commun. 2015; 467(2):223-8. doi: 10.1016/j.bbrc.2015.10.002.

19. Kotake $Y$, Nakagawa T, Kitagawa K, et al. Long non-coding RNA ANRIL is required for the PRC2 recruitment to and silencing of p15(INK4B) tumor suppressor gene. Oncogene. 2011; 30(16):1956-62. doi: 10.1038/onc.2010.568.

20. Xie D, Zhang $\mathrm{H}, \mathrm{Hu} \mathrm{X}$, et al. Knockdown of long non-coding RNA Taurine Up-Regulated 1 inhibited doxorubicin resistance of bladder urothelial carcinoma via Wnt/ $\beta$-catenin pathway. Oncotarget. 2017; 8(51):88689-88696. doi: 10.18632/oncotarget.20927.

21. $\mathrm{Ma} \mathrm{Y}, \mathrm{Hu} \mathrm{X}$, Shang $\mathrm{C}$, et al. Silencing of long non-coding RNA CCAT2 depressed malignancy of oral squamous cell carcinoma via Wnt/ $\beta$-catenin pathway. Tumour Biol. 2017; 39(7):1010428317717670. doi: 10.1177/1010428317717670.

22. Hu B, Zhang H, Wang Z, et al. LncRNA CCAT1/miR-130a-3p axis increases cisplatin resistance in non-small-cell lung cancer cell line by targeting SOX4. Cancer Biol Ther. 2017; 18(12):974-983 doi: 10.1080/15384047 2017.1385679.

23. Zhang $\mathrm{H}$, Guo $\mathrm{Y}$, Song $\mathrm{Y}$, et al. Long noncoding RNA GAS5 inhibits malignant proliferation and chemotherapy resistance to doxorubicin in bladder transitional cell carcinoma. Cancer Chemother Pharmacol. 2017; 79(1):49-55. doi: 10.1007/s00280-016-3194-4.

24. $\mathrm{Xu} \mathrm{R}, \mathrm{Mao} \mathrm{Y}, \mathrm{Chen} \mathrm{K}$, et al. The long noncoding RNA ANRIL acts as an oncogene and contributes to paclitaxel resistance of lung adenocarcinoma A549 cells. Oncotarget. 2017; 8(24):39177-39184. doi: 10.18632/oncotarget.16640.

25. Lan WG, $\mathrm{Xu} \mathrm{DH}, \mathrm{Xu} \mathrm{C}$, et al. Silencing of long non-coding RNA ANRIL inhibits the development of multidrug resistance in gastric cancer cells. Oncol Rep. 2016; 36(1):263-70. doi: 10.3892/or.2016.4771.

26. Wu KF, Liang WC, Feng L, et al. H19 mediates methotrexate resistance in colorectal cancer through activating Wnt/ $\beta$-catenin pathway. Exp Cell Res. 2017; 350(2):312-317. doi: 10.1016/j.yexcr.2016.12.003
27. Zhang H, Wang X, Chen X. Potential Role of Long Non-Coding RNA ANRIL in Pediatric Medulloblastoma Through Promotion on Proliferation and Migration by Targeting miR-323. J Cell Biochem. 2017; 118(12):4735-4744. doi: $10.1002 /$ jcb. 26141.

28. Wang Y, Cheng N, Luo J. Downregulation of lncRNA ANRIL represses tumorigenicity and enhances cisplatin-induced cytotoxicity via regulating microRNA let-7a in nasopharyngeal carcinoma. J Biochem Mol Toxicol. 2017; 31(7). doi: 10.1002/jbt.21904.

29. Jin B, Wang W, Meng XX, et al. Let-7 inhibits self-renewal of hepatocellular cancer stem-like cells through regulating the epithelial-mesenchymal transition and the Wnt signaling pathway. BMC Cancer. 2016; 16(1):863. doi: 10.1186/s12885-016-2904-y 